\title{
Feasibility of a novel mHealth management system to capture and improve medication adherence among adolescents with asthma
}

This article was published in the following Dove Press journal:

Patient Preference and Adherence

4 November 2016

Number of times this article has been viewed

\author{
Anna Cushing ${ }^{1,2}$ \\ Melissa P Manice ${ }^{1,2}$ \\ Andrew Ting ${ }^{3}$ \\ Michael K Parides' \\ 'Department of Population Health \\ Science and Policy, Icahn School \\ of Medicine at Mount Sinai, Icahn \\ Medical Institute, ${ }^{2}$ CoheroHealth, LLC, \\ ${ }^{3}$ Division of Pulmonary and Critical \\ Care, Department of Pediatrics, Icahn \\ School of Medicine at Mount Sinai, \\ New York, NY, USA
}

Purpose: Currently, 7.1 million children in the United States have asthma. Nonadherence to daily controller asthma medication is common, leading to more severe symptoms, overuse of rescue medication, and increased hospitalizations. The purpose of this study was to develop and evaluate the feasibility and acceptability of a novel mHealth management system composed of a sensored device, which is connected to mobile phone app that is designed to monitor and improve asthma medication adherence.

Patients and methods: The asthma management system was designed using well-established behavioral theory. Seven adolescents aged 11-18 years were enrolled and given an adherence sensor, and four of those also received a mobile phone app with game features and reminders Five patients completed the study, and one was lost to follow-up in each group. Mobile app users and their parents participated in focus groups to assess patient preferences. Feasibility was assessed by the ability of sensors to capture real-time medication data. Acceptability was assessed by patient questionnaire and focus group analysis.

Results: Successful upload of real-time data from six of seven inhaler sensors to the HIPAAcompliant server demonstrates the feasibility of at-home patient monitoring using the sensor device. All three mobile app users who completed the study reported interest in continued use of the management system and would recommend the app to friends. Unstructured interviews and focus groups revealed that patients felt that the intervention helped their sense of asthma control.

Conclusion: This study demonstrates the feasibility of using the sensor device to remotely monitor real-time medication usage, and user feedback demonstrates the acceptability of the intervention for patient use. The findings provide guidance for the improvement of study design and technology development. Further research is needed to assess the efficacy of the intervention.

Keywords: asthma control, medication adherence, patient engagement, patient monitoring, mobile health

\section{Introduction}

In the United States, asthma is the single most common chronic disease among children, with 7.1 million children suffering from asthma. ${ }^{1,2}$ The current standard of care for patients with asthma includes a personalized "asthma action plan", a peak flow meter, and prescriptions for a short-acting $\beta$-agonist (albuterol) relief inhaler and long-term controller medication for daily maintenance. ${ }^{3}$ Current adherence rates to daily controller medications for asthma are estimated at 40\%-50\%, though minimum $80 \%$ adherence is required to prevent asthma exacerbations. ${ }^{4-7}$ Thus, many patients do not receive maximum benefit from medical treatment, resulting in poorer health outcomes, lower
Correspondence: Anna Cushing CoheroHealth, 335 Madison Ave, 16th Floor, New York, NY, I0017, USA

Tel +l 6469264370

Email anna.cushing@icahn.mssm.edu 
quality of life, and increased health care costs. ${ }^{8}$ It is estimated that improved patient adherence could prevent $>50 \%$ of the 2.1 million yearly emergency room visits due to asthma and save $>\$ 17$ billion in asthma care costs. ${ }^{8-10}$

Adherence to controller medication is particularly low in younger patients, making them an important target for improvement. ${ }^{5,11}$ In the adolescent population, the most commonly cited reasons for nonadherence are inattention and forgetfulness. ${ }^{12}$ Fortunately, younger patients are the age group who are most motivated and are able to adopt and benefit from mobile technology-based solutions. A 2014 survey of teens in an urban pediatric clinic serving low-income patients in the Bronx found that $80 \%$ owned a smartphone with no difference in smartphone ownership across different household income brackets. ${ }^{13}$ The majority of teens surveyed were interested in using medical smartphone applications, but only $17 \%$ said that they currently use a medical application, demonstrating a clear gap between interest and access to mobile health apps for teens. ${ }^{13}$

A number of studies have recently explored electronic monitoring of asthma medication but do not incorporate proactive patient feedback on medication use, such as responsive message-based reminder functionality. ${ }^{14,15}$ Interventions such as Propeller Health focus on rescue medication use rather than preventative controller medication. ${ }^{16}$ Devices such as the SmartInhaler and SmartTrack have demonstrated increased adherence to inhaled corticosteroids with the use of a sensor that provides audiovisual reminders. ${ }^{17,18}$ However, mobile phones are known to be the preferred engagement platform for reminder cues, rather than the device itself. ${ }^{19,20}$ Visual reminder cues that are tethered to the device have limited utility as a reminder mechanism due to low likelihood that the device is stored in a visible place at the exact moment when the reminder is sent. Beyond short-term studies, reminders alone may not be sufficient to engage patients to have meaningful change in medication use behavior. ${ }^{21-23}$

The design and initial study of a novel management system for asthma described in this study comprised an inhaler sensor to monitor real-time medication adherence and a mobile app to engage patients. The purpose of this study was to assess feasibility and acceptability of this technology in a preliminary study of teens with asthma. The technology is based on the established engagement model of BJ Fogg, which states that behavior change requires ability plus motivation plus trigger. ${ }^{24-26}$ Fogg's model applied to asthma is straightforward: patients need to be equipped with the ability to use medication correctly, motivated by monetary or other incentives, and reminded by well-timed triggers such as text notifications. Thus, this asthma management system can create and reinforce behavior change through smartphones that patients already access on an hourly basis.

\section{Patients and methods Mobile asthma management system}

The inhaler sensor comprises a silicone sleeve that wraps around a standard L-shaped asthma inhaler and a strap that attaches to the cap of the medication dispenser (Figure 1). When medication is dispensed, the sensor records time of medication use through a pressure-sensitive switch and uploads data using Bluetooth low energy to the iPhone app for storage on a Health Insurance Portability and Accountability Act (HIPAA)-compliant server. The sensor devices were preset to allow storage of 50 data points ( $\sim 2$ weeks of data) and upload these data using Bluetooth low energy to the iPhone app, for storage on a secure HIPAA-compliant server.

The mobile app allows patients to view medication usage and sends reminders, with more frequent reminders sent to patients with lower adherence (Figure 2). The app graphically displays daily, weekly, and monthly medication adherence to the patient.

The mobile app engages patients with a customizable "hero" avatar and points redeemable as a gift card of up to $\$ 50$ over the study period, with weekly and monthly bonus points for long-term adherence.

\section{Clinical study design}

English-speaking patients aged 11-19 years were eligible to pilot the inhaler sensor device if they had a diagnosis of persistent asthma and were currently taking a daily asthma controller medication. Patients were required to have access to an iOS device (iPad or iPhone) in order to install and run the mobile app. Patients who were pregnant, in foster care, and emancipated minors were excluded in order to protect these vulnerable populations. A convenience sample of

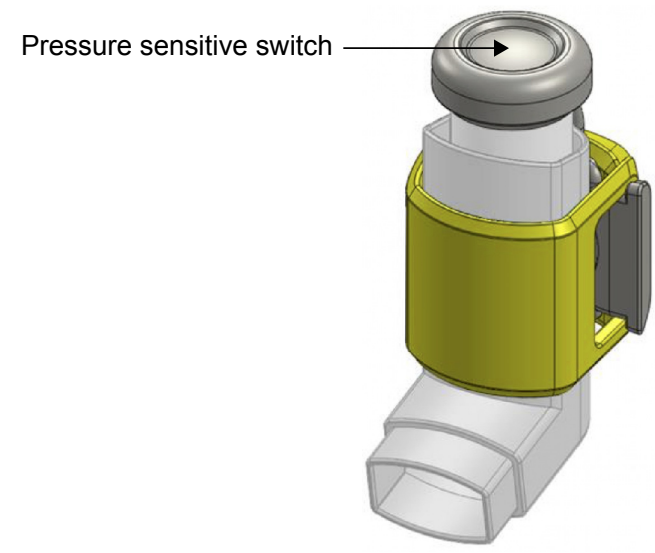

Figure I Inhaler adherence sensor. 


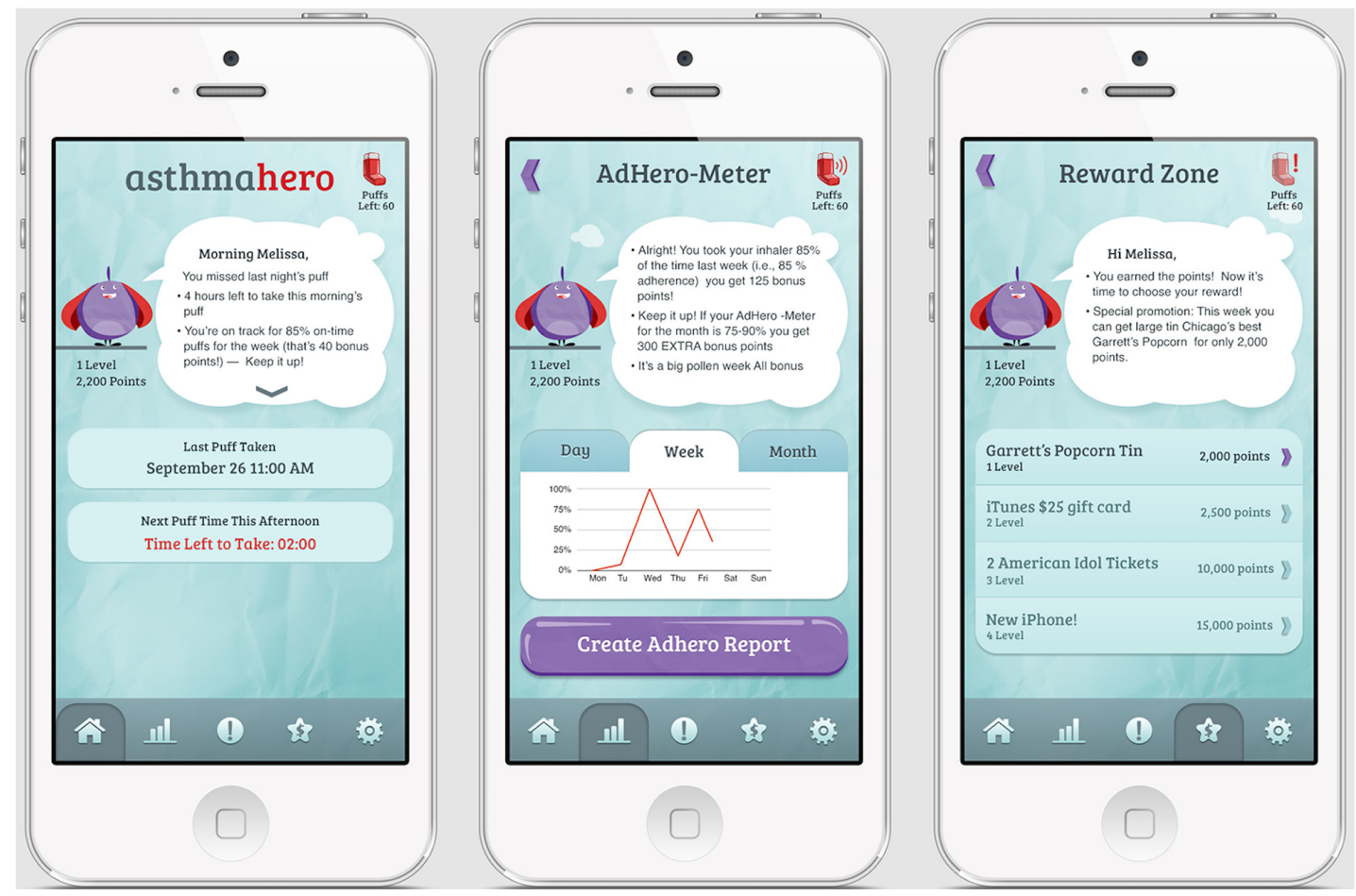

Figure 2 Mobile iPhone app.

Notes: Screenshots of mobile app features, including home screen which displays time of last medication use, graphical display of medication adherence over time which users can download as a PDF Adhero Report, and reward zone where users can redeem gifts for continued medication adherence.

patients were recruited from two Mount Sinai Hospital outpatient pediatric clinics and followed for 12 weeks. Written parental consent and child assent were obtained at enrollment; the study was approved by the Institutional Review Board at Icahn School of Medicine at Mount Sinai. Study patients were assigned to receive the inhaler sensor along with the companion mobile app with reminders and rewards or to receive the inhaler sensor alone with a "control" version of the app designed to facilitate data transfer to the server. All patients participated in baseline and follow-up interviews. At the conclusion of the study, patients in the mobile app users and their parents were interviewed about their experience and were invited to participate in an unstructured interviews or focus groups to discuss their experience with the inhaler sensor and app. Real-time analysis of adherence was not performed due to the small number of patients.

\section{Results}

Seven patients were enrolled to use the adherence sensor, and five completed the study. Four received the adherence sensor with mobile app and three received the adherence sensor only. Baseline characteristics are described in Table 1. At baseline, most patients (6/7) stated that asthma impacted their ability to participate in physical activity, 7/7 reported that sometimes they forgot to take their inhaler, and $5 / 7$ reported that they did not use their daily inhaler when symptoms were under control. Two patients endorsed hospitalizations for asthma in the 12 months prior to the study. Two patients (one from each arm) were lost to follow-up during the study period.

At 12-week follow-up, 4/5 patients reported that asthma impacted their physical activity, $4 / 5$ reported that sometimes they forgot to take their inhaler, and 2/5 reported that they did not use their daily inhaler when symptoms were under control. No study patients reported emergency department visits or hospitalizations for asthma during the study period. All three mobile app users who completed the study agreed that the app helped remind them to take their medication, that they would be interested in continuing to use the mobile app beyond the study period, and that they would recommend the app to other patients suffering from asthma.

Seven sensor devices were used in the study, and six successfully uploaded data to the HIPAA-compliant server. One user's data were not uploaded because the patient was unable to sync the inhaler sensor to his iPad.

Three patients who used the mobile app and inhaler sensor and two of their parents participated in unstructured interviews. Two of the three participants were female, and the patient age ranged from 11 to 14 years. Unstructured interviews led to four main themes: reminders change patient behavior, reminder preferences, influence of a gamified mobile app, and suggestions for hardware improvement. 
Table I Baseline patient characteristics

\begin{tabular}{ll}
\hline Patient characteristics $\mathbf{( N = 7 )}$ & \\
\hline Mean age (range) & 14.1 (II-18) \\
Gender & 2 \\
Male & 5 \\
$\quad$ Female & $823 \mu \mathrm{g}$ \\
Mean daily inhaled corticosteroid dose* & \\
Race & 4 \\
$\quad$ Hispanic & $\mathrm{I}$ \\
White & $\mathrm{I}$ \\
African-American & $\mathrm{I}$ \\
More than one race & \\
Insurance & 2 \\
$\quad$ Private & 5 \\
Medicaid & \\
Household income & \\
$<\$ 25,000$ & 4 \\
$\$ 25,000-\$ 49,000$ & 2 \\
$>\$ 100,000$ & $\mathrm{I}$ \\
\hline
\end{tabular}

Note: *Daily inhaled corticosteroid dose calculated in budesonide propionate equivalents.

Patients reported that they felt the mobile app messages reminded them to take medication that they stated they regularly forgot to take, particularly while taking medication multiple times a day. They described that the mobile app changed their medication use routine and felt that this improved their confidence about asthma control.

Patients described taking their controller medications before leaving for school (before 7-7:30 am) and in the evening after school and after-school activities (after 4-5 pm). They reported receiving multiple reminder messages during the day when they were at school and did not have access to their phones. They agreed that they would like less frequent and shorter messages.

Interviewed patients liked the gaming aspects of the mobile app and felt that the point system and monetary rewards motivated them to use their medication. Two patients suggested adding more features such as unlocking in-app customization of the hero avatar and "mini games" with continued use. The patients reported liking to track their medication adherence and one patient described using the number of points he earned rather than actual medical adherence to follow his progress. One patient reported liking the use of a graph to display trends in adherence, and one patient suggested displaying a number value for adherence rather than a graph.

Two patients noted that the inhaler sensor cap did not stay in place on their inhalers. They found that the flexible cable was too long for their inhalers; hence, the capped part of the sensor would come loose and thus was not always in place when patients used their medication. They recommended customizing the sensor to better fit each individual inhaler type.

\section{Discussion}

This is the first study of the feasibility and acceptability of our novel mHealth asthma management system. Findings provide understanding of feasibility of researching this technology in an adolescent population and acceptability of the intervention to allow for improvements in study design and technology before a large trial is undertaken. Successful uploads of real-time data to the HIPAA-compliant server demonstrate feasibility of at-home patient monitoring using the sensor. One patient who had difficulty syncing the inhaler sensor to his mobile device represents an opportunity to improve installation instructions for usability. Patient questionnaires suggest acceptability of the intervention among the study patients, with all mobile app users expressing interest in continuing to use the mobile app beyond the study period and unstructured interviews, which revealed that patients felt that the mobile app changed their medication use routines, frequency of symptoms, and confidence in their asthma control. Users brought up important factors for improvement such as need to tailor timing of messages in accordance with patients' lifestyles and improve fit and design of the sensor.

Sample size and recruitment were the limitations of this study. The mobile app required patients who have access to an iPhone or iPad. Although smartphones are increasingly prevalent in the teen population across all socioeconomic groups, the requirement that patients specifically have access to an $\mathrm{iOS}$ device may have excluded a number of eligible patient groups. The android version of the mobile app was in development during the course of this study but will be available for use in future studies, and we predict will allow for a more accurate characterization of the adolescent asthma population. The fit of the inhaler sensor was also a limitation of the study as patients reported that it did not always stay on certain types of inhalers during use. This design factor combined with the small sample size of the study precluded accurate real-time data analysis. At the time of publication, redesign of the sensor has already been performed to improve universality of inhaler fit for future studies and has improved patient use.

\section{Conclusion}

These findings provide opportunity for optimization of study design and technology before a larger trial is undertaken. The use of real-time adherence data in a larger patient 
population will allow better assessment of reliability and efficacy of the intervention. Future study design should include clinical metrics for asthma severity such as lung function (forced expiratory volume in 1 second), morbidity, use of rescue medication or oral steroids, and asthma control test.

\section{Acknowledgment}

Sudha Busani aided in implementation and primary data collection of the patient study.

\section{Disclosure}

Dr Manice is the CEO of CoheroHealth, the company that developed the mobile intervention and sponsored the study, and reports no other conflicts of interest in this work. Anna Cushing is a part-time employee of CoheroHealth and a full-time student, and reports no other conflicts of interest in this work. Dr Ting and Dr Parides report no conflicts of interest in this work.

\section{References}

1. World Health Organization. Asthma Fact Sheet; 2013. Available from: http://www.who.int/mediacentre/factsheets/fs307/en/. Accessed November 3, 2014.

2. CDC. FastStats - asthma. Available from: http://www.cdc.gov/nchs/ fastats/asthma.htm. Accessed October 14, 2014.

3. NHLBI. Asthma Care Quick Reference: Diagnosing and Managing Asthma. Available from: http://www.nhlbi.nih.gov/health-pro/ guidelines/current/asthma-guidelines/quick-reference-html.htm. Accessed January 8, 2015.

4. Lasmar L, Camargos P, Champs NS, et al. Adherence rate to inhaled corticosteroids and their impact on asthma control. Allergy. 2009;64(5): 784-789.

5. Bender BG, Rand C. Medication non-adherence and asthma treatment cost. Curr Opin Allergy Clin Immunol. 2004;4(3):191-195.

6. Claxton AJ, Cramer J, Pierce C. A systematic review of the associations between dose regimens and medication compliance. Clin Ther. 2001;23(8):1296-1310.

7. Lasmar L, Camargos P, Bousquet J, Goulart E, Sakurai E, Carvalhais M. Factors related to lower adherence rates to inhaled corticosteroids in children and adolescents: a prospective randomized cohort study. J Trop Pediatr. 2009;55(1):20-25.

8. Barnett SB, Nurmagambetov TA. Costs of asthma in the United States: 2002-2007. J Allergy Clin Immunol. 2011;127(1):145-152.
9. Barnes P, Jonsson B, Klim J. The costs of asthma. Eur Respir J. 1996;9(4): 636-642.

10. Weiss KB, Sullivan SD. The health economics of asthma and rhinitis. I. Assessing the economic impact. J Allergy Clin Immunol. 2001; 107(1):3-8.

11. Einarson TR. Drug-related hospital admissions. Ann Pharmacother. 1993;27(7-8):832-840.

12. Chapman KR, Walker L, Cluley S, Fabbri L. Improving patient compliance with asthma therapy. Respir Med. 2000;94(1):2-9.

13. Singh A, Wilkinson S, Braganza S. Smartphones and pediatric apps to mobilize the medical home. J Pediatr. 2014;165(3):606-610.

14. Van Sickle D, Magzamen S, Truelove S, Morrison T. Remote monitoring of inhaled bronchodilator use and weekly feedback about asthma management: an open-group, short-term pilot study of the impact on asthma control. PLoS One. 2013;8(2):e55335.

15. Julius SM, Sherman JM, Hendeles L. Accuracy of three electronic monitors for metered-dose inhalers. Chest. 2002;121(3):871-876.

16. Merchant RK, Inamdar R, Quade RC. Effectiveness of population health management using the propeller health asthma platform: a randomized clinical trial. J Allergy Clin Immunol Pract. 2016;4(3):455-463.

17. Patel M, Pilcher J, Travers J, et al. Use of metered-dose inhaler electronic monitoring in a real-world asthma randomized controlled trial. J Allergy Clin Immunol Pract. 2013;1(1):83-91.

18. Foster JM, Smith L, Usherwood T, Sawyer SM, Rand CS, Reddel HK. The reliability and patient acceptability of the SmartTrack device: a new electronic monitor and reminder device for metered dose inhalers. J Asthma. 2012;49(6):657-662.

19. Patrick H, Williams GC. Self-determination theory: its application to health behavior and complementarity with motivational interviewing. Int J Behav Nutr Phys Act. 2012;9(18):1479-5868.

20. Heimlich JE, Ardoin NM. Understanding behavior to understand behavior change: a literature review. Environ Educ Res. 2008;14(3): 215-237.

21. Tran N, Fau CJ, Fau SK, Cabana M. Patient reminder systems and asthma medication adherence: a systematic review. J Asthma. 2014;51(5): 536-543.

22. Nundy S, Mishra A, Hogan P, Lee SM, Solomon MC, Peek ME. How do mobile phone diabetes programs drive behavior change? Evidence from a mixed methods observational cohort study. Diabetes Educ. 2014; 40(6):806-819.

23. Vodopivec-Jamsek V, de Jongh T, Gurol-Urganci I, Atun R, Car J. Mobile phone messaging for preventive health care. Cochrane Database Syst Rev. 2012;12:CD007457.

24. Fogg B. A behavior model for persuasive design. Proceedings of the 4th International Conference on Persuasive Technology; 2009; Claremont, CA.

25. King D, Greaves F, Exeter C, Darzi A. "Gamification": influencing health behaviours with games. J Royal Soc Med. 2013;106(3):76-78.

26. Lin RJ, Zhu X. Leveraging social media for preventive care - a gamification system and insights. Stud Health Technol Inform. 2012; $180: 838-842$.
Patient Preference and Adherence

\section{Publish your work in this journal}

Patient Preference and Adherence is an international, peer-reviewed, open access journal that focuses on the growing importance of patient preference and adherence throughout the therapeutic continuum. Patient satisfaction, acceptability, quality of life, compliance, persistence and their role in developing new therapeutic modalities and compounds to optimize

\section{Dovepress}

clinical outcomes for existing disease states are major areas of interest for the journal. This journal has been accepted for indexing on PubMed Central. The manuscript management system is completely online and includes a very quick and fair peer-review system, which is all easy to use. Visit http://www. dovepress.com/testimonials.php to read real quotes from published authors. 